\title{
Sex/Gender/Sexual Science Research 24/7
}

\author{
Kenneth J. Zucker ${ }^{1}$
}

Published online: 29 March 2018

(c) Springer Science+Business Media, LLC, part of Springer Nature 2018

Since my last Editorial (Zucker, 2016), it feels that the Journal has ramped up in terms of "busyness." When I became Editor in 2002, we had 80 new submissions. There has been, more or less, a linear increase since then, hitting a milestone of sorts in 2017, when we surpassed 500 new submissions for the first time (Fig. 1). I don't think that this temporal pattern is unique to Archives: Its fraternal opposite-sex twin, Journal of Sex Research, had 477 new submissions in 2017 (C. A. Graham, personal communication, January 1, 2018). Along with dozens of resubmissions, Letters to the Editor, and commentaries on the new Target Article series, the pace has a relentless, yet rewarding, feel to it. One reason for the incremental increase in submissions is that sex/gender/sexual science research has truly gone global (cf. Zucker, 2002). We now routinely receive submissions from all corners of the planet. Maybe one of these days we will get a submission from the International Space Station.

\section{Associate Editors}

Back in 2010, when the number of submissions was clearly on the rise $(N=295)$, it became apparent to me that the Journal needed to add Associate Editors, which took place in 2011. The Journal's boss, Carol Bischoff, at Springer Nature has been very generous in this regard and, at present, we have 15 Associate Editors (Sophie Bergeron, Lori Brotto, Meredith Chivers, Brian Dodge, Shari Dworkin, Lisa Dawn Hamilton, Roland Imhoff, Tuuli Kukkonen, Brian Mustanski, Kevin Nunes, Jeffrey Parsons, Natalie Rosen, Alexander Schmidt, Lori Scott-Sheldon, and Paul Vasey). These scholars all have their own speciality areas and handle submissions that match their areas of expertise. I handle about $50 \%$ of the new submissions myself. We also have, by and large, a wonderful Editorial Board currently

Kenneth J. Zucker

ken.zucker@utoronto.ca

1 Department of Psychiatry, University of Toronto, Toronto, ON M5T 1R8, Canada totaling 139 , not to mention the dozens of ad hoc reviewers who are also asked to review manuscripts by me and the Associate Editors. It is this collaborative, collective, communal process that keeps the sexual science engine running.

\section{New Editorial Board Members (2018)}

These are: Steven Arnocky, Ally L. Dir, Breanne Fahs, Brian A. Feinstein, Kyle Gobrogge, Lisette Kuyper, Kristen Mark, Bill McCarthy, Andreas Mokros, Sarah A. Vannier, and Julia Velten.

\section{New Look (2018)}

Earlier this year, Springer Nature has given the Journal, like all of its other periodicals, a new font face. In addition, the Abstract spans the entire width of the page instead of being in double columns. I like the changes.

\section{Manuscript Disposition (2015-2016)}

Table 1 shows Editorial decision data for initial submissions for 2015 and 2016 as a function of who handled them (2017 data are not yet available) (see also Fig. 2). Table 1 does not include 23 manuscripts handled by Vasey for a Special Section (Vasey, 2017), which were by invitation. From Table 1, it can be seen that the percentage of "rejected" manuscripts was $57.8 \%$ in 2015 and $53.6 \%$ in 2016. It can also be seen that there was variability in the percentage of declined submissions across the "handler." Of course, this could reflect variation in the standards of the Editor/Associate Editors and the reviewers assigned to the manuscripts; however, it is my view that most of the variance is accounted for by the quality of submissions, which varies across specialty areas. For contributing authors, it is important to note that when manuscripts are given the designation of a Major Revision, the vast majority (of those that are resubmitted) are accepted for publication (it is quite 
Fig. 1 Number of submissions (2012-2017)

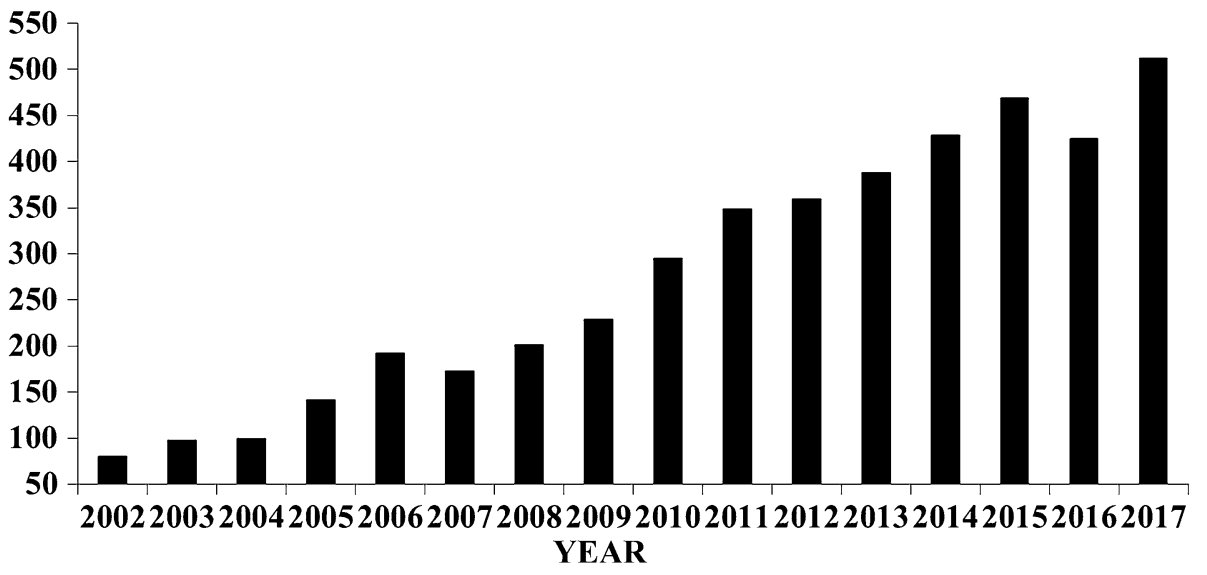

Table 1 Editorial decisions for initial submissions (2015 and 2016)

\begin{tabular}{|c|c|c|c|}
\hline $\begin{array}{l}\text { Editor/Associate } \\
\text { Editors }\end{array}$ & $\begin{array}{l}\text { Accept/ } \\
\text { Provisional } \\
\text { Accept }\end{array}$ & Major Revision & Reject \\
\hline Bergeron & $\begin{array}{l}0 \\
0\end{array}$ & $\begin{array}{l}7 \\
6\end{array}$ & $\begin{array}{l}10(58.8 \%) \\
12(66.6 \%)\end{array}$ \\
\hline Bradford & $\begin{array}{l}2 \\
-\end{array}$ & $\begin{array}{l}2 \\
-\end{array}$ & $\begin{array}{l}19(85.1 \%) \\
-\end{array}$ \\
\hline Brotto & $\begin{array}{l}0 \\
1\end{array}$ & $\begin{array}{l}6 \\
5\end{array}$ & $\begin{array}{l}7(53.8 \%) \\
10(62.5 \%)\end{array}$ \\
\hline Chivers & $\begin{array}{l}0 \\
0\end{array}$ & $\begin{array}{l}7 \\
4\end{array}$ & $\begin{array}{l}4(36.3 \%) \\
1(20.0 \%)\end{array}$ \\
\hline Dodge & $\begin{array}{l}1 \\
0\end{array}$ & $\begin{array}{l}13 \\
7\end{array}$ & $\begin{array}{l}3(17.6 \%) \\
6(46.1 \%)\end{array}$ \\
\hline Dworkin & $\begin{array}{l}6 \\
7\end{array}$ & $\begin{array}{l}15 \\
9\end{array}$ & $\begin{array}{l}29(58.0 \%) \\
31(65.9 \%)\end{array}$ \\
\hline Lalumiére & $\begin{array}{l}3 \\
3\end{array}$ & $\begin{array}{l}2 \\
6\end{array}$ & $\begin{array}{l}16(76.1 \%) \\
8(47.0 \%)\end{array}$ \\
\hline Mustanski & $\begin{array}{l}0 \\
0\end{array}$ & $\begin{array}{l}8 \\
9\end{array}$ & $\begin{array}{l}10(55.5 \%) \\
6(40.0 \%)\end{array}$ \\
\hline Owen/Hamilton & $\begin{array}{l}0 \\
1\end{array}$ & $\begin{array}{l}1 \\
2\end{array}$ & $\begin{array}{l}11(91.6 \%) \\
6(66.6 \%)\end{array}$ \\
\hline Parsons & $\begin{array}{l}2 \\
4\end{array}$ & $\begin{array}{l}8 \\
7\end{array}$ & $\begin{array}{l}7(41.1 \%) \\
8(42.1 \%)\end{array}$ \\
\hline Rosen & $\begin{array}{l}0 \\
1\end{array}$ & $\begin{array}{l}1 \\
3\end{array}$ & $\begin{array}{l}0(0.0 \%) \\
16(80.0 \%)\end{array}$ \\
\hline Scott-Sheldon & $\begin{array}{l}2 \\
0\end{array}$ & $\begin{array}{l}9 \\
10\end{array}$ & $\begin{array}{l}20(64.5 \%) \\
14(58.3 \%)\end{array}$ \\
\hline Zucker & $\begin{array}{l}19 \\
20\end{array}$ & $\begin{array}{l}82 \\
83\end{array}$ & $\begin{array}{l}133(56.8 \%) \\
100(49.2 \%)\end{array}$ \\
\hline Total & $\begin{array}{l}35 \\
37\end{array}$ & $\begin{array}{l}161 \\
151\end{array}$ & $\begin{array}{l}269(57.8 \%) \\
218(53.6 \%)\end{array}$ \\
\hline
\end{tabular}

Data for 2015 are on the first row, and data for 2016 are on the second row. Associate Editor Hamilton began in 2017, but handled one submission from 2016, so is combined with Owen. Associate Editor Rosen began in 2016, but handled one submission from 2015

uncommon for a Major Revision to be declined after resubmission-to quote a colleague "It happens," but not very often). This means that the initial decision indicates that the author has a "foot in the door" and that if she, he, or they can successfully respond to the first round of reviews it means that the manuscript is very likely to be accepted for publication.

\section{Target Articles}

Under Paul Vasey's leadership, we inaugurated the by invitation Target Article series (Vasey \& Zucker, 2016). To date, we have published 7 such articles (Blanchard, 2018; Breedlove, 2017; Brotto \& Yule, 2017; Chivers, 2017; Frank, 2018; Seto, 2017; Walton, Cantor, Bhullar, \& Lykins, 2017). Each Target Article is accompanied by penetrating commentaries, which are also reviewed by Vasey, with a response by the author(s). More are in the sexual science pipeline. I expect these Target Articles will make a contribution to the Journal's impact factor-we will find out in 2019. Stay tuned. Researchers interested in proposing a Target Article should contact Dr. Vasey at paul.vasey@uleth.ca

\section{Impact Factor (2015 and 2016)}

Tables 2, 3, 4, and 5 show the impact factor (IF) data for the years 2015 and 2016 for 86 sex/gender journals in the Annual Web of Science/Journal Citation Reports (Clarivate Analytics). ${ }^{1}$ Tables 2 and 4 rank the journals as a function of their 2 -year IF, and Tables 3 and 5 rank the journals as a function of their 5-year IF.

For the uninitiated to this treasure trove of numbers, a journal's IF for a given year is a measure of the frequency with which its recent articles are cited on average during that year.

\footnotetext{
1 There are other sex/gender journals that do not have Impact Factor data (e.g., International Journal of Transgenderism, Journal of Black Sexuality and Relationships, Journal of Language \& Sexuality, Partner Abuse, Porn Studies, Psychology of Sexual Orientation \& Gender Diversity, Psychology \& Sexuality, Sexualization, Media, \& Society, Transgender Health, Transgender Studies Quarterly, and Violence and Gender) (see also https ://en.wikipedia.org/wiki/List_of_sexology_journals).
} 
Fig. 2 Manuscript disposition after initial submission (2002-2016)

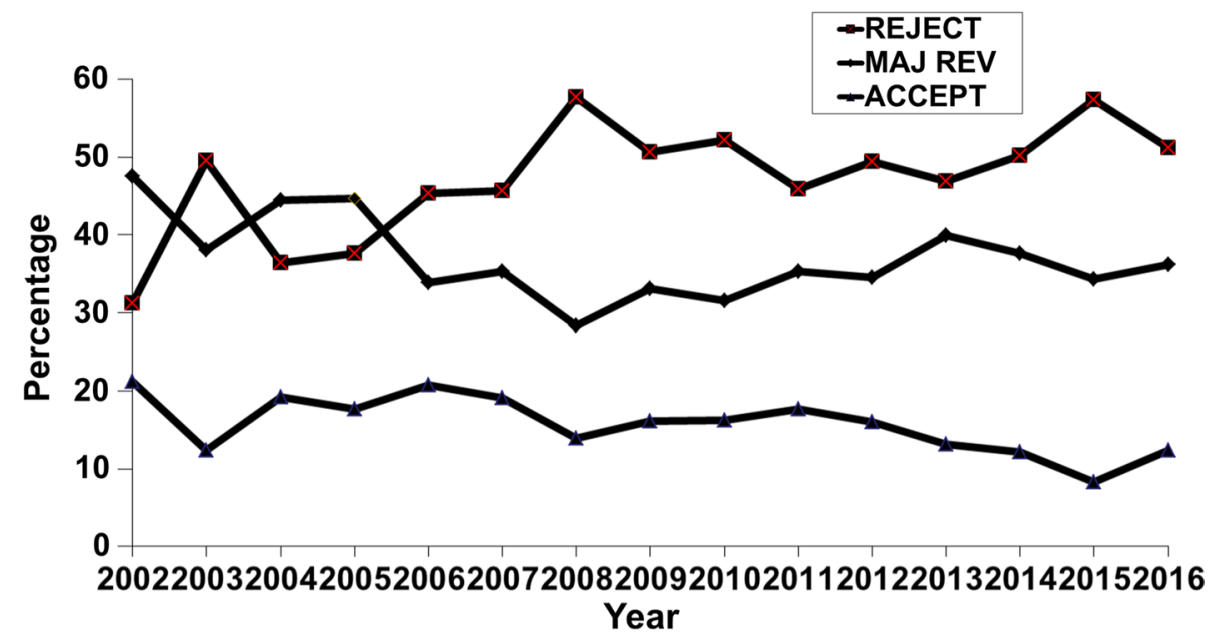

"Recent" refers to the two prior calendar years or the five prior calendar years. Thus, Archives' 2015 2-year IF is the number of times that its 2013 and 2014 articles were cited in 2015, divided by the number of articles the Archives published in 2013 and 2014, and the 5-year IF is the number of times that its 2010-2014 articles were cited in 2015, divided by the number of articles the Archives published between 2010 and 2014. The 5-year IF was introduced several years ago, and one can find data on it going back to 2007. Although the IF is the best known metric for citation analysis, there are other measures, including the immediacy index (II) and the cited half-Life (CHL). The II is a measure of how frequently the journal's "average article" is cited the same year in which it is published. Thus, the II for a year is calculated as the number of times articles from that journal are cited during that year, divided by the number of articles that journal published that year. The CHL is a measure of the longevity of the frequency of citations to articles in the journal, that is, for how long the average article maintains its currency. The CHL for a year is determined by the time required to account for a cumulative total of $50 \%$ of that year's citations to the journal.

In these two calendar years, over 3000 journals in the Social Sciences received IF ratings. In 2015-2016, across all of these journals, the median IF ranged from 1.00 to 1.27 (across the 2-year and 5-year rankings). During these 2 years, we published 351 articles ("Citable Items") and Archives ranked between the 87 th and 90th percentiles, which is not bad. Two years ago (Zucker, 2016), I indicated a greater fondness for the 5-year IF than the 2-year. I still feel that way. I hope that will be the case this year!

How "stable" is the IF among the sex/gender periodicals? As a cursory look at this, I examined the IF comparing the years 2012 and 2016. For 80 periodicals for which there were data from both years, a paired samples $t$ test showed that the mean IF was significantly higher in $2016(M, 1.75$; $\mathrm{SD}=1.15)$ than in $2012(M, 1.47 ; \mathrm{SD}=1.31), t(79)=4.44, p<.001$, Cohen's $d=.54$. The two scores were, however, highly correlated at $r=.92, p<.001$. Rank ordering the periodicals yielded an $r=.90, p<.001$. These numbers suggest a fair bit of stability. Out of curiosity, I looked at a few of the periodicals that had the most "movement" between 2012 and 2016 (based on the 80 periodicals for which there were data from both years). On the positive side, Perspectives on Sexual and Reproductive Health ranked 35th in 2012 and 6th in 2016; differences: A Journal of Feminist Cultural Studies ranked 71st in 2012 and 42nd in 2016; and Feminist Review ranked 63rd in 2012 and 41st in 2016. On the negative side, Evolutionary Psychology ranked 23rd in 2012 and 49th in 2016; Journal of Men's Health ranked 47th in 2012 and 71st in 2016; and Sexual Health ranked 27th in 2012 and 50th in 2016.

I must confess that I had never heard of the IF until I heard my old pal, A. Koos Slob, from Erasmus University in Rotterdam shout out in a dimly lit bar at some meeting of the International Academy of Sex Research (a long time ago, maybe 1995 in Provincetown) "What's the impact factor?" I almost choked on my White Russian. Although there are many criticisms of the IF, we know that it is taken seriously (e.g., for promotions at universities or medical centers). Of course, every Editor worth her, his or they's salt thinks about how their IF can be improved. It is well known (from many bar room convos) that one unnamed sex/gender periodical for a time "padded" its IF by asking authors to cite publications from the journal, even if the publications were completely irrelevant to the content of the paper. But one has to be careful with too many "self cites" because this is monitored by Journal Citation Reports and, if there are too many, a journal can get into trouble. So, are there ways to legitimately improve a journal's IF? I am sure that there are. It has been argued that review articles and special topics can be contributory. I know, for example, that when this Journal published the literature reviews from the Sexual and Gender Identity Disorders Workgroup for the DSM-5 in 2010 it had a clear impact (Zucker, 2010). As noted earlier, it is my hope that the introduction of the Target Article series will be one bona fide way in which the IF might be improved. 
Table 2 Impact Factor for the year 2015: Sex- and gender-related journals $(N=86)$ ranked by 2-year impact factor

\begin{tabular}{|c|c|c|c|c|c|c|}
\hline Journal & $\begin{array}{l}2 \text {-year } \\
\text { Impact } \\
\text { Factor }\end{array}$ & $\begin{array}{l}\text { 5-year } \\
\text { Impact } \\
\text { Factor }\end{array}$ & $\begin{array}{l}2015 \text { Total } \\
\text { Cites }\end{array}$ & 2015 articles & Immediacy Index & $\begin{array}{l}\text { Cited Half- } \\
\text { Life }\end{array}$ \\
\hline Archives of Sexual Behavior & 2.704 & 3.440 & 5016 & 180 & 0.600 & 6.5 \\
\hline Psychoneuroendocrinology & 4.704 & 5.183 & 13,117 & 269 & 1.093 & 6.4 \\
\hline Human Reproduction & 4.621 & 4.635 & 27,878 & 307 & 1.199 & 9.4 \\
\hline Fertility and Sterility & 4.426 & 4.333 & 31,421 & 418 & 0.916 & 7.3 \\
\hline AIDS & 4.407 & 4.784 & 20,134 & 272 & 1.026 & 7.3 \\
\hline Journal of Acquired Immune Deficiency Syndromes & 3.806 & 3.953 & 13,282 & 341 & 1.076 & 6.2 \\
\hline Hormones and Behavior & 3.340 & 3.923 & 9163 & 150 & 0.847 & 7.8 \\
\hline Biology of Sex Differences & 3.237 & & 344 & 34 & 0.235 & 3.3 \\
\hline Evolution and Human Behavior & 3.223 & 3.687 & 3285 & 64 & 0.781 & 8.0 \\
\hline Perspectives on Sexual and Reproductive Health & 3.214 & 3.224 & 1234 & 20 & 0.300 & 7.6 \\
\hline Menopause & 3.172 & 3.019 & 4632 & 161 & 1.174 & 5.3 \\
\hline AIDS and Behavior & 3.063 & 3.467 & 6847 & 251 & 0.566 & 4.5 \\
\hline AIDS Patient Care and STDs & 3.035 & 3.410 & 3035 & 81 & 0.691 & 6.1 \\
\hline Sexually Transmitted Infections & 3.015 & 2.915 & 4598 & 102 & 1.255 & 6.6 \\
\hline Sexually Transmitted Diseases & 2.968 & 3.014 & 5526 & 123 & 0.561 & 7.3 \\
\hline Psychology of Men \& Masculinity & 2.947 & 3.116 & 1259 & 43 & 1.023 & 6.2 \\
\hline Journal of Sex Research & 2.862 & 3.274 & 3055 & 86 & 0.384 & $>10.0$ \\
\hline Journal of Sexual Medicine & 2.844 & 2.884 & 7994 & 262 & 0.698 & 5.1 \\
\hline Asian Journal of Andrology & 2.664 & 2.579 & 2619 & 130 & 1.046 & 4.5 \\
\hline Andrology US & 2.515 & & 676 & 133 & 0.564 & 2.0 \\
\hline Gender and Society & 2.400 & 3.265 & 2979 & 36 & 0.583 & $>10.0$ \\
\hline Psychology of Women Quarterly & 2.397 & 2.278 & 2706 & 36 & 0.500 & $>10.0$ \\
\hline LGBT Health & 2.261 & & 112 & 48 & 0.167 & 1.5 \\
\hline Sexual Development & 2.164 & 1.928 & 585 & 31 & 0.548 & 5.0 \\
\hline Body Image & 2.104 & 2.804 & 2124 & 71 & 1.225 & 5.8 \\
\hline Journal of Women's Health & 2.032 & 2.270 & 3920 & 121 & 0.397 & 5.9 \\
\hline $\begin{array}{l}\text { International Perspectives on Sexual and Reproduc- } \\
\text { tive Health }\end{array}$ & 2.030 & 2.000 & 295 & 19 & 0.579 & 4.3 \\
\hline Sexual Abuse: A Journal of Research and Treatment & 1.981 & 2.938 & 1023 & 35 & 0.200 & 9.4 \\
\hline AIDS Care & 1.902 & 2.147 & 5490 & 222 & 0.441 & 6.4 \\
\hline Women's Health Issues & 1.811 & 2.307 & 1490 & 91 & 0.308 & 5.4 \\
\hline American Journal of Men's Health & 1.713 & 1.551 & 511 & 50 & 0.380 & 3.6 \\
\hline Hormone Research in Pediatrics & 1.661 & 1.778 & 1147 & 109 & 0.284 & 3.8 \\
\hline Culture, Health, and Sexuality & 1.588 & 1.754 & 1494 & 91 & 0.385 & 5.4 \\
\hline Sex Roles & 1.582 & 1.801 & 5566 & 76 & 0.553 & $>10.0$ \\
\hline AIDS Education and Prevention & 1.524 & 2.163 & 1735 & 40 & 0.225 & 9.2 \\
\hline Journal of Sex \& Marital Therapy & 1.514 & 1.867 & 1764 & 53 & 0.302 & $>10.0$ \\
\hline Sexuality Research and Social Policy & 1.452 & 1.575 & 437 & 33 & 0.273 & 5.4 \\
\hline International Journal of Impotence Research & 1.396 & 1.484 & 1932 & 45 & 0.133 & $>10.0$ \\
\hline BMC Women's Health & 1.353 & 1.636 & 817 & 119 & 0.168 & 4.3 \\
\hline Signs & 1.347 & 1.302 & 1805 & 39 & 0.359 & $>10.0$ \\
\hline Women \& Health & 1.337 & 1.562 & 1252 & 56 & 0.107 & 9.8 \\
\hline Gender, Work, and Organization & 1.325 & 2.021 & 1089 & 37 & 0.189 & 8.2 \\
\hline International Journal of STD and AIDS & 1.300 & 1.211 & 2688 & 164 & 0.360 & 6.8 \\
\hline Feminism and Psychology & 1.290 & 1.308 & 665 & 46 & 0.457 & 8.5 \\
\hline Feminist Theory & 1.268 & 1.194 & 419 & 16 & 0.250 & 7.9 \\
\hline Men and Masculinities & 1.250 & 1.885 & 681 & 26 & 0.385 & $>10.0$ \\
\hline Sexual Health & 1.225 & 1.493 & 961 & 72 & 0.431 & 4.3 \\
\hline
\end{tabular}


Table 2 (continued)

\begin{tabular}{|c|c|c|c|c|c|c|}
\hline Journal & $\begin{array}{l}\text { 2-year } \\
\text { Impact } \\
\text { Factor }\end{array}$ & $\begin{array}{l}\text { 5-year } \\
\text { Impact } \\
\text { Factor }\end{array}$ & $\begin{array}{l}2015 \text { Total } \\
\text { Cites }\end{array}$ & 2015 articles & Immediacy Index & $\begin{array}{l}\text { Cited Half- } \\
\text { Life }\end{array}$ \\
\hline Sexual \& Reproductive Healthcare & 1.211 & 1.424 & 206 & 44 & 0.159 & 3.5 \\
\hline Sexual Medicine & 1.188 & & 47 & 30 & 0.200 & \\
\hline Gender, Place, and Culture & 1.180 & 1.656 & 1026 & 76 & 0.211 & $>10.0$ \\
\hline Body \& Society & 1.171 & 2.373 & 733 & 19 & 0.263 & 9.4 \\
\hline European Journal of Women's Studies & 1.160 & 1.209 & 418 & 30 & 0.167 & 8.6 \\
\hline Feminist Review & 1.156 & 1.032 & 513 & 14 & 0.429 & $>10.0$ \\
\hline Sexual and Relationship Therapy & 1.109 & 1.109 & 521 & 33 & 0.219 & 8.0 \\
\hline $\begin{array}{l}\text { Journal of Psychosomatic Obstetrics and Gynecol- } \\
\text { ogy }\end{array}$ & 1.065 & 1.962 & 1055 & 20 & 0.050 & 9.8 \\
\hline Evolutionary Psychology & 1.050 & 1.383 & 609 & 51 & 0.118 & 5.6 \\
\hline Feminist Criminology & 1.032 & 1.519 & 220 & 16 & & 6.4 \\
\hline Politics and Gender & 1.024 & 1.652 & 367 & 27 & 0.111 & 7.0 \\
\hline Violence Against Women & 1.020 & 1.858 & 2333 & 72 & 0.250 & 8.8 \\
\hline Gender and Education & 0.905 & 1.151 & 846 & 50 & & $>10.0$ \\
\hline Journal of Homosexuality & 0.862 & 1.518 & 1898 & 89 & 0.143 & 9.9 \\
\hline Sexuality and Disability & 0.771 & 1.069 & 488 & 38 & 0.053 & 8.9 \\
\hline Feminist Legal Studies & 0.731 & 1.143 & 201 & 12 & 2.167 & 6.1 \\
\hline International Journal of Sexual Health & 0.729 & 1.033 & 200 & 37 & 0.081 & 4.9 \\
\hline Journal of Sexual Aggression & 0.723 & 0.992 & 267 & 25 & 0.080 & 6.5 \\
\hline African Journal of AIDS Research & 0.716 & 0.866 & 333 & 38 & 0.026 & 6.1 \\
\hline Zeitschrift für Sexualforschung & 0.690 & 0.521 & 82 & 20 & 0.300 & \\
\hline Sexualities & 0.673 & 1.018 & 711 & 51 & 0.078 & 8.1 \\
\hline Journal of Men's Health & 0.652 & 0.753 & 194 & & & 4.7 \\
\hline Journal of Gender Studies & 0.604 & 0.904 & 298 & 39 & 0.154 & 7.3 \\
\hline Women's Studies International Forum & 0.591 & 0.751 & 882 & 83 & 0.084 & $>10.0$ \\
\hline Journal of Child Sexual Abuse & 0.536 & 1.090 & 581 & 51 & 0.167 & 6.8 \\
\hline Feminist Studies & 0.520 & 0.546 & 600 & 26 & 0.038 & $>10.0$ \\
\hline Hypatia: A Journal of Feminist Philosophy & 0.519 & 0.804 & 746 & 51 & 0.255 & $>10.0$ \\
\hline Sex Education & 0.505 & & 301 & 47 & 0.191 & 6.3 \\
\hline Australian Feminist Studies & 0.500 & 0.507 & 150 & 12 & & 6.2 \\
\hline $\begin{array}{l}\text { International Journal of Feminist Approaches to } \\
\text { Bioethics }\end{array}$ & 0.488 & & 90 & 16 & 0.062 & \\
\hline GLQ: A Journal of Gay and Lesbian Studies & 0.333 & 0.956 & 397 & 23 & 0.048 & $>10.0$ \\
\hline differences: A Journal of Feminist Cultural Studies & 0.310 & 0.606 & 262 & 23 & 0.261 & $>10.0$ \\
\hline Gender and Language & 0.267 & 0.521 & 64 & 16 & 0.188 & \\
\hline Indian Journal of Gender Studies & 0.231 & 0.301 & 88 & 21 & & \\
\hline Women and Therapy & 0.229 & 0.221 & 207 & 23 & & $>10.0$ \\
\hline Frontiers: Journal of Women's Studies & 0.214 & 0.224 & 147 & 30 & 0.067 & $>10.0$ \\
\hline Asian Journal of Women's Studies & 0.214 & 0.216 & 41 & 29 & 0.040 & \\
\hline Journal of the History of Sexuality & 0.121 & 0.293 & 203 & 16 & 0.062 & $>10.0$ \\
\hline Feministische Studien & 0.077 & 0.063 & 25 & 14 & & \\
\hline
\end{tabular}

Total cites indicates the total number of times that each journal has been cited by all journals included in the ISI database in 2015. Immediacy index means cites in 2015 to articles published in 2015/number of articles published in 2015. Cited half-life is the median age of its articles cited in the current year. Half of the citations to the journal are to articles published within the cited half-life. Of 3211 Social Science journals, Behavioral and Brain Sciences had the highest 2-year impact factor (20.415). Of 3233 Life Sciences journals, Cancer Journal for Clinicians had the highest 2-year impact factor (137.57) 
Table 3 Impact Factor for the year 2015: Sex- and gender-related journals $(N=86)$ ranked by 5-year impact factor

\begin{tabular}{|c|c|c|c|c|c|c|}
\hline Journal & $\begin{array}{l}\text { 2-year } \\
\text { Impact } \\
\text { Factor }\end{array}$ & $\begin{array}{l}\text { 5-year } \\
\text { Impact } \\
\text { Factor }\end{array}$ & $\begin{array}{l}2015 \text { Total } \\
\text { Cites }\end{array}$ & 2015 articles & Immediacy Index & $\begin{array}{l}\text { Cited Half- } \\
\text { Life }\end{array}$ \\
\hline Archives of Sexual Behavior & 2.704 & 3.440 & 5016 & 180 & 0.600 & 6.5 \\
\hline Psychoneuroendocrinology & 4.704 & 5.183 & 13,117 & 269 & 1.093 & 6.4 \\
\hline AIDS & 4.407 & 4.784 & 20,134 & 272 & 1.026 & 7.3 \\
\hline Human Reproduction & 4.621 & 4.635 & 27,878 & 307 & 1.199 & 9.4 \\
\hline Fertility and Sterility & 4.426 & 4.333 & 31,421 & 418 & 0.916 & 7.3 \\
\hline Journal of Acquired Immune Deficiency Syndromes & 3.806 & 3.953 & 13,282 & 341 & 1.076 & 6.2 \\
\hline Hormones and Behavior & 3.340 & 3.923 & 9163 & 150 & 0.847 & 7.8 \\
\hline Evolution and Human Behavior & 3.223 & 3.687 & 3285 & 64 & 0.781 & 8.0 \\
\hline AIDS and Behavior & 3.063 & 3.467 & 6847 & 251 & 0.566 & 4.5 \\
\hline AIDS Patient Care and STDs & 3.035 & 3.410 & 3035 & 81 & 0.691 & 6.1 \\
\hline Journal of Sex Research & 2.862 & 3.274 & 3055 & 86 & 0.384 & $>10.0$ \\
\hline Gender and Society & 2.400 & 3.265 & 2979 & 36 & 0.583 & $>10.0$ \\
\hline Perspectives on Sexual and Reproductive Health & 3.214 & 3.224 & 1234 & 20 & 0.300 & 7.6 \\
\hline Psychology of Men \& Masculinity & 2.947 & 3.116 & 1259 & 43 & 1.023 & 6.2 \\
\hline Menopause & 3.172 & 3.019 & 4632 & 161 & 1.174 & 5.3 \\
\hline Sexual Abuse: A Journal of Research and Treatment & 1.981 & 2.938 & 1023 & 35 & 0.200 & 9.4 \\
\hline Sexually Transmitted Infections & 3.015 & 2.915 & 4598 & 102 & 1.255 & 6.6 \\
\hline Sexually Transmitted Diseases & 2.968 & 3.014 & 5526 & 123 & 0.561 & 7.3 \\
\hline Journal of Sexual Medicine & 2.844 & 2.884 & 7994 & 262 & 0.698 & 5.1 \\
\hline Body Image & 2.104 & 2.804 & 2124 & 71 & 1.225 & 5.8 \\
\hline Asian Journal of Andrology & 2.664 & 2.579 & 2619 & 130 & 1.046 & 4.5 \\
\hline Body \& Society & 1.171 & 2.373 & 733 & 19 & 0.263 & 9.4 \\
\hline Women's Health Issues & 1.811 & 2.307 & 1490 & 91 & 0.308 & 5.4 \\
\hline Psychology of Women Quarterly & 2.397 & 2.278 & 2706 & 36 & 0.500 & $>10.0$ \\
\hline Journal of Women's Health & 2.032 & 2.270 & 3920 & 121 & 0.397 & 5.9 \\
\hline AIDS Education and Prevention & 1.524 & 2.163 & 1735 & 40 & 0.225 & 9.2 \\
\hline AIDS Care & 1.902 & 2.147 & 5490 & 222 & 0.441 & 6.4 \\
\hline Gender, Work, and Organization & 1.325 & 2.021 & 1089 & 37 & 0.189 & 8.2 \\
\hline $\begin{array}{l}\text { International Perspectives on Sexual and Reproduc- } \\
\text { tive Health }\end{array}$ & 2.030 & 2.000 & 295 & 19 & 0.579 & 4.3 \\
\hline $\begin{array}{l}\text { Journal of Psychosomatic Obstetrics and Gynecol- } \\
\text { ogy }\end{array}$ & 1.065 & 1.962 & 1055 & 20 & 0.050 & 9.8 \\
\hline Sexual Development & 2.164 & 1.928 & 585 & 31 & 0.548 & 5.0 \\
\hline Men and Masculinities & 1.250 & 1.885 & 681 & 26 & 0.385 & $>10.0$ \\
\hline Journal of Sex \& Marital Therapy & 1.514 & 1.867 & 1764 & 53 & 0.302 & $>10.0$ \\
\hline Violence Against Women & 1.020 & 1.858 & 2333 & 72 & 0.250 & 8.8 \\
\hline Sex Roles & 1.582 & 1.801 & 5566 & 76 & 0.553 & $>10.0$ \\
\hline Hormone Research in Pediatrics & 1.661 & 1.778 & 1147 & 109 & 0.284 & 3.8 \\
\hline Culture, Health, and Sexuality & 1.588 & 1.754 & 1494 & 91 & 0.385 & 5.4 \\
\hline Gender, Place, and Culture & 1.180 & 1.656 & 1026 & 76 & 0.211 & $>10.0$ \\
\hline Politics and Gender & 1.024 & 1.652 & 367 & 27 & 0.111 & 7.0 \\
\hline BMC Women's Health & 1.353 & 1.636 & 817 & 119 & 0.168 & 4.3 \\
\hline Sexuality Research and Social Policy & 1.452 & 1.575 & 437 & 33 & 0.273 & 5.4 \\
\hline Women \& Health & 1.337 & 1.562 & 1252 & 56 & 0.107 & 9.8 \\
\hline American Journal of Men's Health & 1.713 & 1.551 & 511 & 50 & 0.380 & 3.6 \\
\hline Feminist Criminology & 1.032 & 1.519 & 220 & 16 & & 6.4 \\
\hline Journal of Homosexuality & 0.862 & 1.518 & 1898 & 89 & 0.143 & 9.9 \\
\hline Sexual Health & 1.225 & 1.493 & 961 & 72 & 0.431 & 4.3 \\
\hline
\end{tabular}


Table 3 (continued)

\begin{tabular}{|c|c|c|c|c|c|c|}
\hline Journal & $\begin{array}{l}\text { 2-year } \\
\text { Impact } \\
\text { Factor }\end{array}$ & $\begin{array}{l}\text { 5-year } \\
\text { Impact } \\
\text { Factor }\end{array}$ & $\begin{array}{l}2015 \text { Total } \\
\text { Cites }\end{array}$ & 2015 articles & Immediacy Index & $\begin{array}{l}\text { Cited Half- } \\
\text { Life }\end{array}$ \\
\hline International Journal of Impotence Research & 1.396 & 1.484 & 1932 & 45 & 0.133 & $>10.0$ \\
\hline Sexual \& Reproductive Healthcare & 1.211 & 1.424 & 206 & 44 & 0.159 & 3.5 \\
\hline Evolutionary Psychology & 1.050 & 1.383 & 609 & 51 & 0.118 & 5.6 \\
\hline Feminism and Psychology & 1.290 & 1.308 & 665 & 46 & 0.457 & 8.5 \\
\hline Signs & 1.347 & 1.302 & 1805 & 39 & 0.359 & $>10.0$ \\
\hline International Journal of STD and AIDS & 1.300 & 1.211 & 2688 & 164 & 0.360 & 6.8 \\
\hline European Journal of Women's Studies & 1.160 & 1.209 & 418 & 30 & 0.167 & 8.6 \\
\hline Feminist Theory & 1.268 & 1.194 & 419 & 16 & 0.250 & 7.9 \\
\hline Gender and Education & 0.905 & 1.151 & 846 & 50 & & $>10.0$ \\
\hline Feminist Legal Studies & 0.731 & 1.143 & 201 & 12 & 2.167 & 6.1 \\
\hline Sexual and Relationship Therapy & 1.109 & 1.109 & 521 & 33 & 0.219 & 8.0 \\
\hline Journal of Child Sexual Abuse & 0.536 & 1.090 & 581 & 51 & 0.167 & 6.8 \\
\hline Sexuality and Disability & 0.771 & 1.069 & 488 & 38 & 0.053 & 8.9 \\
\hline International Journal of Sexual Health & 0.729 & 1.033 & 200 & 37 & 0.081 & 4.9 \\
\hline Feminist Review & 1.156 & 1.032 & 513 & 14 & 0.429 & $>10.0$ \\
\hline Sexualities & 0.673 & 1.018 & 711 & 51 & 0.078 & 8.1 \\
\hline Journal of Sexual Aggression & 0.723 & 0.992 & 267 & 25 & 0.080 & 6.5 \\
\hline GLQ: A Journal of Gay and Lesbian Studies & 0.333 & 0.956 & 397 & 23 & 0.048 & $>10.0$ \\
\hline Journal of Gender Studies & 0.604 & 0.904 & 298 & 39 & 0.154 & 7.3 \\
\hline African Journal of AIDS Research & 0.716 & 0.866 & 333 & 38 & 0.026 & 6.1 \\
\hline Hypatia: A Journal of Feminist Philosophy & 0.519 & 0.804 & 746 & 51 & 0.255 & $>10.0$ \\
\hline Journal of Men's Health & 0.652 & 0.753 & 194 & & & 4.7 \\
\hline Women's Studies International Forum & 0.591 & 0.751 & 882 & 83 & 0.084 & $>10.0$ \\
\hline differences: A Journal of Feminist Cultural Studies & 0.310 & 0.606 & 262 & 23 & 0.261 & $>10.0$ \\
\hline Feminist Studies & 0.520 & 0.546 & 600 & 26 & 0.038 & $>10.0$ \\
\hline Gender and Language & 0.267 & 0.521 & 64 & 16 & 0.188 & \\
\hline Zeitschrift für Sexualforschung & 0.690 & 0.521 & 82 & 20 & 0.300 & \\
\hline Australian Feminist Studies & 0.500 & 0.507 & 150 & 12 & & 6.2 \\
\hline Indian Journal of Gender Studies & 0.231 & 0.301 & 88 & 21 & & \\
\hline Journal of the History of Sexuality & 0.121 & 0.293 & 203 & 16 & 0.062 & $>10.0$ \\
\hline Frontiers: Journal of Women's Studies & 0.214 & 0.224 & 147 & 30 & 0.067 & $>10.0$ \\
\hline Women and Therapy & 0.229 & 0.221 & 207 & 23 & & $>10.0$ \\
\hline Asian Journal of Women's Studies & 0.214 & 0.216 & 41 & 29 & 0.040 & \\
\hline Feministische Studien & 0.077 & 0.063 & 25 & 14 & & \\
\hline Biology of Sex Differences & 3.237 & & 344 & 34 & 0.235 & 3.3 \\
\hline Andrology US & 2.515 & & 676 & 133 & 0.564 & 2.0 \\
\hline LGBT Health & 2.261 & & 112 & 48 & 0.167 & 1.5 \\
\hline Sexual Medicine & 1.188 & & 47 & 30 & 0.200 & \\
\hline Sex Education & 0.505 & & 301 & 47 & 0.191 & 6.3 \\
\hline $\begin{array}{l}\text { International Journal of Feminist Approaches to } \\
\text { Bioethics }\end{array}$ & 0.488 & & 90 & 16 & 0.062 & \\
\hline
\end{tabular}

Total cites indicates the total number of times that each journal has been cited by all journals included in the ISI database in 2015 . Immediacy index means cites in 2015 to articles published in 2015/number of articles published in 2015. Cited half-life is the median age of its articles cited in the current year. Half of the citations to the journal are to articles published within the cited half-life 
Table 4 Impact Factor for the year 2016: Sex- and gender-related journals $(N=86)$ ranked by 2-year impact factor

\begin{tabular}{|c|c|c|c|c|c|c|}
\hline Journal & $\begin{array}{l}\text { 2-year } \\
\text { Impact } \\
\text { Factor }\end{array}$ & $\begin{array}{l}\text { 5-year } \\
\text { Impact } \\
\text { Factor }\end{array}$ & $\begin{array}{l}2016 \text { Total } \\
\text { Cites }\end{array}$ & 2016 articles & Immediacy Index & $\begin{array}{l}\text { Cited Half- } \\
\text { Life }\end{array}$ \\
\hline Archives of Sexual Behavior & 2.720 & 3.217 & 5849 & 171 & 0.515 & 6.9 \\
\hline Human Reproduction & 5.020 & 5.008 & 29,721 & 305 & 1.102 & 9.6 \\
\hline AIDS & 5.003 & 4.971 & 20,962 & 286 & 1.367 & 7.7 \\
\hline Psychoneuroendocrinology & 4.788 & 5.381 & 14,409 & 325 & 0.843 & 6.5 \\
\hline Fertility and Sterility & 4.373 & 4.595 & 33,098 & 437 & 1.050 & 7.7 \\
\hline Journal of Acquired Immune Deficiency Syndromes & 3.935 & 3.879 & 14,067 & 315 & 1.137 & 6.5 \\
\hline Biology of Sex Differences & 3.635 & - & 571 & 68 & - & 3.7 \\
\hline Perspectives on Sexual and Reproductive Health & 3.571 & 3.178 & 1169 & 20 & 0.400 & 8.2 \\
\hline Evolution and Human Behavior & 3.383 & 3.863 & 3651 & 58 & 1.000 & 8.3 \\
\hline Hormones and Behavior & 3.378 & 4.033 & 9447 & 149 & 0.799 & $>10.0$ \\
\hline AIDS Patient Care and STDs & 3.236 & 3.459 & 3187 & 65 & 0.400 & 6.4 \\
\hline Sexually Transmitted Infections & 3.212 & 3.045 & 4626 & 106 & 1.255 & 7.1 \\
\hline Asian Journal of Andrology & 2.996 & 2.796 & 3118 & 135 & 1.674 & 4.7 \\
\hline Journal of Sexual Medicine & 2.978 & 3.095 & 9411 & 181 & 0.729 & 5.6 \\
\hline Body Image & 2.926 & 3.175 & 2602 & 92 & 0.489 & 6.2 \\
\hline Sexual Abuse: A Journal of Research and Treatment & 2.926 & 2.909 & 1271 & 35 & 0.200 & 9.4 \\
\hline AIDS and Behavior & 2.916 & 3.264 & 7248 & 309 & 0.566 & 4.8 \\
\hline Journal of Sex Research & 2.902 & 3.450 & 3474 & 92 & 0.435 & $>10.0$ \\
\hline Gender and Society & 2.765 & 3.540 & 3689 & 39 & 0.282 & $>10.0$ \\
\hline Menopause & 2.733 & 2.860 & 4783 & 166 & 0.783 & 5.8 \\
\hline Psychology of Women Quarterly & 2.432 & 3.049 & 2943 & 39 & 1.026 & $>10.0$ \\
\hline Andrology & 2.427 & 2.487 & 1000 & 110 & 0.682 & 2.5 \\
\hline Sexually Transmitted Diseases & 2.358 & 2.699 & 5526 & 139 & 0.345 & 8.0 \\
\hline Journal of Women's Health & 2.322 & 2.546 & 4186 & 147 & 0.259 & 6.3 \\
\hline American Journal of Men's Health & 2.141 & 1.813 & 669 & 71 & 0.366 & 4.0 \\
\hline Politics and Gender & 2.109 & 2.773 & 634 & 37 & 0.108 & $>10.0$ \\
\hline LGBT Health & 2.053 & 2.191 & 238 & 53 & 0.604 & 2.2 \\
\hline Sexual Development & 1.972 & 2.069 & 737 & 33 & 0.515 & 5.9 \\
\hline Psychology of Men \& Masculinity & 1.957 & 2.387 & 1127 & 37 & 0.324 & 7.2 \\
\hline Sex Roles & 1.954 & 2.616 & 6837 & 87 & 0.770 & $>10.0$ \\
\hline Body \& Society & 1.951 & 2.104 & 899 & 21 & 0.095 & 9.5 \\
\hline Culture, Health, and Sexuality & 1.951 & 2.059 & 1881 & 100 & 0.210 & 5.7 \\
\hline Journal of Sex \& Marital Therapy & 1.935 & 2.205 & 1973 & 48 & 0.208 & $>10.0$ \\
\hline Hormone Research in Pediatrics & 1.844 & 1.819 & 1352 & 104 & 0.356 & 3.9 \\
\hline AIDS Care & 1.824 & 2.073 & 5504 & 305 & 0.410 & 6.9 \\
\hline Women's Health Issues & 1.801 & 2.308 & 1682 & 80 & 0.512 & 5.7 \\
\hline $\begin{array}{l}\text { International Perspectives on Sexual and Reproduc- } \\
\text { tive Health }\end{array}$ & 1.686 & 2.437 & 329 & 8 & & 4.8 \\
\hline Sexual Medicine & 1.683 & 1.681 & 122 & 30 & 0.033 & 2.3 \\
\hline AIDS Education and Prevention & 1.671 & 1.927 & 1744 & 42 & 0.167 & 9.9 \\
\hline Gender, Place, and Culture & 1.605 & 1.856 & 1306 & 119 & 0.261 & $>10.0$ \\
\hline $\begin{array}{l}\text { Journal of Psychosomatic Obstetrics and Gynecol- } \\
\text { ogy }\end{array}$ & 1.575 & 2.134 & 1165 & 20 & 0.200 & 9.7 \\
\hline BMC Women’s Health & 1.572 & 1.791 & 1076 & 72 & 0.153 & 4.0 \\
\hline Gender, Work, and Organization & 1.548 & 2.067 & 1302 & 38 & 0.211 & 8.2 \\
\hline Sexuality Research and Social Policy & 1.547 & 1.694 & 597 & 34 & 0.588 & 6.2 \\
\hline Feminist Review & 1.519 & 1.135 & 595 & 16 & 0.062 & $>10.0$ \\
\hline differences: A Journal of Feminist Cultural Studies & 1.467 & 1.257 & 385 & 20 & 0.200 & 9.4 \\
\hline
\end{tabular}


Table 4 (continued)

\begin{tabular}{|c|c|c|c|c|c|c|}
\hline Journal & $\begin{array}{l}\text { 2-year } \\
\text { Impact } \\
\text { Factor }\end{array}$ & $\begin{array}{l}\text { 5-year } \\
\text { Impact } \\
\text { Factor }\end{array}$ & $\begin{array}{l}2016 \text { Total } \\
\text { Cites }\end{array}$ & 2016 articles & Immediacy Index & $\begin{array}{l}\text { Cited Half- } \\
\text { Life }\end{array}$ \\
\hline Women \& Health & 1.437 & 1.746 & 1342 & 58 & 0.086 & 9.9 \\
\hline Violence Against Women & 1.423 & 2.155 & 3150 & 88 & 0.284 & 9.5 \\
\hline Journal of Homosexuality & 1.369 & 1.827 & 2221 & 89 & 0.135 & $>10.0$ \\
\hline International Journal of STD and AIDS & 1.350 & 1.258 & 2773 & 188 & 0.340 & 6.9 \\
\hline Men and Masculinities & 1.308 & 1.823 & 747 & 25 & 0.080 & 8.4 \\
\hline International Journal of Impotence Research & 1.293 & 1.450 & 2123 & 44 & 0.295 & 9.4 \\
\hline Evolutionary Psychology & 1.243 & 1.552 & 687 & 40 & 0.150 & 5.1 \\
\hline Sexual Health & 1.238 & 1.298 & 903 & 88 & 0.261 & 4.8 \\
\hline Sexual \& Reproductive Healthcare & 1.208 & 1.680 & 312 & 50 & 0.160 & 3.9 \\
\hline European Journal of Women's Studies & 1.132 & 1.452 & 544 & 22 & 0.091 & $>10.0$ \\
\hline Feminist Legal Studies & 1.130 & 1.306 & 247 & 14 & 2.143 & 6.9 \\
\hline Sexual and Relationship Therapy & 1.188 & 1.101 & 521 & 33 & 0.30 & 8.7 \\
\hline Feminist Theory & 1.114 & 1.956 & 545 & 18 & 0.333 & $>10.0$ \\
\hline Journal of Sexual Aggression & 1.082 & 1.168 & 362 & 29 & 0.103 & 6.9 \\
\hline Hypatia: A Journal of Feminist Philosophy & 1.038 & 1.168 & 1053 & 53 & 0.057 & $>10.0$ \\
\hline Feminist Criminology & 0.970 & 1.159 & 315 & 15 & 0.267 & $>10.0$ \\
\hline Sexualities & 0.922 & 1.251 & 862 & 48 & 0.083 & 8.4 \\
\hline Sexuality and Disability & 0.908 & 1.121 & 562 & 32 & 0.188 & 8.5 \\
\hline Signs & 0.906 & 1.532 & 2283 & 38 & 0.342 & $>10.0$ \\
\hline African Journal of AIDS Research & 0.861 & 1.112 & 424 & 41 & 0.220 & 5.2 \\
\hline GLQ: A Journal of Gay and Lesbian Studies & 0.861 & 0.730 & 452 & 23 & 0.261 & 7.5 \\
\hline International Journal of Sexual Health & 0.852 & 1.053 & 248 & 33 & 0.121 & 8.8 \\
\hline Australian Feminist Studies & 0.810 & 0.733 & 252 & 26 & 0.077 & 5.9 \\
\hline Feminism and Psychology & 0.757 & 1.312 & 781 & 28 & 0.857 & $>10.0$ \\
\hline Sex Education & 0.753 & - & 467 & 47 & 0.149 & 5.2 \\
\hline Women's Studies International Forum & 0.686 & 0.885 & 1099 & 59 & 0.102 & 7.5 \\
\hline Journal of Gender Studies & 0.676 & 1.080 & 335 & 46 & 0.065 & 8.8 \\
\hline Journal of Child Sexual Abuse & 0.649 & 1.080 & 719 & 51 & 0.098 & 5.9 \\
\hline Gender and Education & 0.639 & 0.931 & 897 & 56 & 0.107 & $>10.0$ \\
\hline Journal of Men's Health & 0.516 & 0.762 & 206 & 6 & - & $>10.0$ \\
\hline $\begin{array}{l}\text { International Journal of Feminist Approaches to } \\
\text { Bioethics }\end{array}$ & 0.462 & 0.739 & 104 & N/A & N/A & 7.4 \\
\hline Gender \& Language & 0.406 & 0.641 & 64 & 19 & 0.053 & 9.6 \\
\hline Zeitschrift für Sexualforschung & 0.382 & 0.408 & 63 & 13 & 0.231 & - \\
\hline Frontiers: Journal of Women's Studies & 0.379 & 0.475 & 170 & 22 & - & 9.00 \\
\hline Feminist Studies & 0.353 & 0.531 & 719 & 25 & 0.080 & $>10.0$ \\
\hline Women and Therapy & 0.348 & 0.508 & 326 & 36 & 0.056 & $>10.0$ \\
\hline Asian Journal of Women's Studies & 0.321 & 0.270 & 63 & 29 & 0.034 & - \\
\hline Journal of the History of Sexuality & 0.250 & 0.455 & 241 & 16 & 0.062 & $>10.0$ \\
\hline Indian Journal of Gender Studies & 0.150 & 0.330 & 122 & 21 & 0.143 & 8.3 \\
\hline Feministische Studien & 0.107 & 0.102 & 54 & 14 & - & - \\
\hline
\end{tabular}

Total cites indicates the total number of times that each journal has been cited by all journals included in the ISI database in 2016 . Immediacy index means cites in 2016 to articles published in 2016/number of articles published in 2016. Cited half-life is the median age of its articles cited in the current year. Half of the citations to the journal are to articles published within the cited half-life

Of 3236 Social Science journals, World Psychiatry had the highest 2-year impact factor (26.56). Of 8856 Life Sciences journals, Cancer Journal for Clinicians had the highest 2-year impact factor (187.04) 
Table 5 Impact factor for the year 2016: Sex- and gender-related journals $(N=86)$ ranked by 5-year impact factor

\begin{tabular}{|c|c|c|c|c|c|c|}
\hline Journal & $\begin{array}{l}\text { 2-year } \\
\text { Impact } \\
\text { Factor }\end{array}$ & $\begin{array}{l}\text { 5-year } \\
\text { Impact } \\
\text { Factor }\end{array}$ & $\begin{array}{l}2016 \text { Total } \\
\text { Cites }\end{array}$ & 2016 articles & Immediacy Index & $\begin{array}{l}\text { Cited Half- } \\
\text { Life }\end{array}$ \\
\hline Archives of Sexual Behavior & 2.720 & 3.217 & 5849 & 171 & 0.515 & 6.9 \\
\hline Psychoneuroendocrinology & 4.788 & 5.381 & 14,409 & 325 & 0.843 & 6.5 \\
\hline Human Reproduction & 5.020 & 5.008 & 29,721 & 305 & 1.102 & 9.6 \\
\hline AIDS & 5.003 & 4.971 & 20,962 & 286 & 1.367 & 7.7 \\
\hline Fertility and Sterility & 4.373 & 4.595 & 33,098 & 437 & 1.050 & 7.7 \\
\hline Hormones and Behavior & 3.378 & 4.033 & 9447 & 149 & 0.799 & $>10.0$ \\
\hline Journal of Acquired Immune Deficiency Syndromes & 3.935 & 3.879 & 14,067 & 315 & 1.137 & 6.5 \\
\hline Evolution and Human Behavior & 3.383 & 3.863 & 3651 & 58 & 1.000 & 8.3 \\
\hline Gender and Society & 2.765 & 3.540 & 3689 & 39 & 0.282 & $>10.0$ \\
\hline AIDS Patient Care and STDs & 3.236 & 3.459 & 3187 & 65 & 0.400 & 6.4 \\
\hline Journal of Sex Research & 2.902 & 3.450 & 3474 & 92 & 0.435 & $>10.0$ \\
\hline AIDS and Behavior & 2.916 & 3.264 & 7248 & 309 & 0.566 & 4.8 \\
\hline Perspectives on Sexual and Reproductive Health & 3.571 & 3.178 & 1169 & 20 & 0.400 & 8.2 \\
\hline Body Image & 2.926 & 3.175 & 2602 & 92 & 0.489 & 6.2 \\
\hline Journal of Sexual Medicine & 2.978 & 3.095 & 9411 & 181 & 0.729 & 5.6 \\
\hline Psychology of Women Quarterly & 2.432 & 3.049 & 2943 & 39 & 1.026 & $>10.0$ \\
\hline Sexually Transmitted Infections & 3.212 & 3.045 & 4626 & 106 & 1.255 & 7.1 \\
\hline Sexual Abuse: A Journal of Research and Treatment & 2.926 & 2.909 & 1271 & 35 & 0.200 & 9.4 \\
\hline Menopause & 2.733 & 2.860 & 4783 & 166 & 0.783 & 5.8 \\
\hline Asian Journal of Andrology & 2.996 & 2.796 & 3118 & 135 & 1.674 & 4.7 \\
\hline Politics and Gender & 2.109 & 2.773 & 634 & 37 & 0.108 & $>10.0$ \\
\hline Sexually Transmitted Diseases & 2.358 & 2.699 & 5526 & 139 & 0.345 & 8.0 \\
\hline Sex Roles & 1.954 & 2.616 & 6837 & 87 & 0.770 & $>10.0$ \\
\hline Journal of Women's Health & 2.322 & 2.546 & 4186 & 147 & 0.259 & 6.3 \\
\hline Andrology & 2.427 & 2.487 & 1000 & 110 & 0.682 & 2.5 \\
\hline $\begin{array}{l}\text { International Perspectives on Sexual and Reproduc- } \\
\text { tive Health }\end{array}$ & 1.686 & 2.437 & 329 & 8 & & 4.8 \\
\hline Psychology of Men \& Masculinity & 1.957 & 2.387 & 1127 & 37 & 0.324 & 7.2 \\
\hline Women's Health Issues & 1.801 & 2.308 & 1682 & 80 & 0.512 & 5.7 \\
\hline Journal of Sex \& Marital Therapy & 1.935 & 2.205 & 1973 & 48 & 0.208 & $>10.0$ \\
\hline LGBT Health & 2.053 & 2.191 & 238 & 53 & 0.604 & 2.2 \\
\hline Violence Against Women & 1.423 & 2.155 & 3150 & 88 & 0.284 & 9.5 \\
\hline $\begin{array}{l}\text { Journal of Psychosomatic Obstetrics and Gynecol- } \\
\text { ogy }\end{array}$ & 1.575 & 2.134 & 1165 & 20 & 0.200 & 9.7 \\
\hline Body \& Society & 1.951 & 2.104 & 899 & 21 & 0.095 & 9.5 \\
\hline AIDS Care & 1.824 & 2.073 & 5504 & 305 & 0.410 & 6.9 \\
\hline Sexual Development & 1.972 & 2.069 & 737 & 33 & 0.515 & 5.9 \\
\hline Gender, Work, and Organization & 1.548 & 2.067 & 1302 & 38 & 0.211 & 8.2 \\
\hline Culture, Health, and Sexuality & 1.951 & 2.059 & 899 & 21 & 0.095 & 9.5 \\
\hline Feminist Theory & 1.114 & 1.956 & 545 & 18 & 0.333 & $>10.0$ \\
\hline AIDS Education and Prevention & 1.671 & 1.927 & 1744 & 42 & 0.167 & 9.9 \\
\hline Gender, Place, and Culture & 1.605 & 1.856 & 1306 & 119 & 0.261 & $>10.0$ \\
\hline Journal of Homosexuality & 1.369 & 1.827 & 2221 & 89 & 0.135 & $>10.0$ \\
\hline Men and Masculinities & 1.308 & 1.823 & 747 & 25 & 0.080 & 8.4 \\
\hline Hormone Research in Pediatrics & 1.844 & 1.819 & 1352 & 104 & 0.356 & 3.9 \\
\hline American Journal of Men's Health & 2.141 & 1.813 & 669 & 71 & 0.366 & 4.0 \\
\hline BMC Women's Health & 1.572 & 1.791 & 1076 & 72 & 0.153 & 4.0 \\
\hline Women \& Health & 1.437 & 1.746 & 1342 & 58 & 0.086 & 9.9 \\
\hline
\end{tabular}


Table 5 (continued)

\begin{tabular}{|c|c|c|c|c|c|c|}
\hline Journal & $\begin{array}{l}\text { 2-year } \\
\text { Impact } \\
\text { Factor }\end{array}$ & $\begin{array}{l}\text { 5-year } \\
\text { Impact } \\
\text { Factor }\end{array}$ & $\begin{array}{l}2016 \text { Total } \\
\text { Cites }\end{array}$ & 2016 articles & Immediacy Index & $\begin{array}{l}\text { Cited Half- } \\
\text { Life }\end{array}$ \\
\hline Sexuality Research and Social Policy & 1.547 & 1.694 & 597 & 34 & 0.588 & 6.2 \\
\hline Sexual Medicine & 1.683 & 1.681 & 122 & 30 & 0.033 & 2.3 \\
\hline Sexual \& Reproductive Healthcare & 1.208 & 1.680 & 312 & 50 & 0.160 & 3.9 \\
\hline Evolutionary Psychology & 1.243 & 1.552 & 687 & 40 & 0.150 & 5.1 \\
\hline Signs & 0.906 & 1.532 & 2283 & 38 & 0.342 & $>10.0$ \\
\hline International Journal of Impotence Research & 1.293 & 1.450 & 2123 & 44 & 0.295 & 9.4 \\
\hline European Journal of Women’s Studies & 1.132 & 1.452 & 544 & 22 & 0.091 & $>10.0$ \\
\hline Feminism and Psychology & 0.757 & 1.312 & 781 & 28 & 0.857 & $>10.0$ \\
\hline Feminist Legal Studies & 1.130 & 1.306 & 247 & 14 & 2.143 & 6.9 \\
\hline Sexual Health & 1.238 & 1.298 & 903 & 88 & 0.261 & 4.8 \\
\hline International Journal of STD and AIDS & 1.350 & 1.258 & 2773 & 188 & 0.340 & 6.9 \\
\hline differences: A Journal of Feminist Cultural Studies & 1.467 & 1.257 & 385 & 20 & 0.200 & 9.4 \\
\hline Sexualities & 0.922 & 1.251 & 862 & 48 & 0.083 & 8.4 \\
\hline Hypatia: A Journal of Feminist Philosophy & 1.038 & 1.168 & 1053 & 53 & 0.057 & $>10.0$ \\
\hline Journal of Sexual Aggression & 1.082 & 1.168 & 362 & 29 & 0.103 & 6.9 \\
\hline Feminist Criminology & 0.970 & 1.159 & 315 & 15 & 0.267 & $>10.0$ \\
\hline Feminist Review & 1.519 & 1.135 & 595 & 16 & 0.062 & $>10.0$ \\
\hline Sexuality and Disability & 0.908 & 1.121 & 562 & 32 & 0.188 & 8.5 \\
\hline African Journal of AIDS Research & 0.861 & 1.112 & 424 & 41 & 0.220 & 5.2 \\
\hline Sexual and Relationship Therapy & 1.188 & 1.101 & 521 & 33 & 0.30 & 8.7 \\
\hline Journal of Child Sexual Abuse & 0.649 & 1.080 & 719 & 51 & 0.098 & 5.9 \\
\hline Journal of Gender Studies & 0.676 & 1.080 & 335 & 46 & 0.065 & 8.8 \\
\hline International Journal of Sexual Health & 0.852 & 1.053 & 248 & 33 & 0.121 & 8.8 \\
\hline Gender and Education & 0.639 & 0.931 & 897 & 56 & 0.107 & $>10.0$ \\
\hline Women's Studies International Forum & 0.686 & 0.885 & 1099 & 59 & 0.102 & 7.5 \\
\hline Hypatia: A Journal of Feminist Philosophy & 0.519 & 0.804 & 746 & 51 & 0.255 & $>10.0$ \\
\hline Journal of Men's Health & 0.516 & 0.762 & 206 & 6 & - & $>10.0$ \\
\hline $\begin{array}{l}\text { International Journal of Feminist Approaches to } \\
\text { Bioethics }\end{array}$ & 0.462 & 0.739 & 104 & N/A & N/A & 7.4 \\
\hline Australian Feminist Studies & 0.810 & 0.733 & 252 & 26 & 0.077 & 5.9 \\
\hline GLQ: A Journal of Gay and Lesbian Studies & 0.861 & 0.730 & 452 & 23 & 0.261 & 7.5 \\
\hline Gender \& Language & 0.406 & 0.641 & 64 & 19 & 0.053 & 9.6 \\
\hline Feminist Studies & 0.353 & 0.531 & 719 & 25 & 0.080 & $>10.0$ \\
\hline Women and Therapy & 0.348 & 0.508 & 326 & 36 & 0.056 & $>10.0$ \\
\hline Frontiers: Journal of Women's Studies & 0.379 & 0.475 & 170 & 22 & - & 9.00 \\
\hline Journal of the History of Sexuality & 0.250 & 0.455 & 241 & 16 & 0.062 & $>10.0$ \\
\hline Zeitschrift für Sexualforschung & 0.382 & 0.408 & 63 & 13 & 0.231 & - \\
\hline Indian Journal of Gender Studies & 0.150 & 0.330 & 122 & 21 & 0.143 & 8.3 \\
\hline Asian Journal of Women's Studies & 0.321 & 0.270 & 63 & 29 & 0.034 & - \\
\hline Feministische Studien & 0.107 & 0.102 & 54 & 14 & - & - \\
\hline Biology of Sex Differences & 3.635 & - & & & & \\
\hline Sex Education & 0.753 & - & & & & \\
\hline
\end{tabular}

Total cites indicates the total number of times that each journal has been cited by all journals included in the ISI database in 2016 . Immediacy index means cites in 2016 to articles published in 2016/number of articles published in 2016. Cited half-life is the median age of its articles cited in the current year. Half of the citations to the journal are to articles published within the cited half-life. Of 3236 Social Science journals, World Psychiatry had the highest 2-year impact factor (26.56). Of 8856 Life Sciences journals, Cancer Journal for Clinicians had the highest 2-year impact factor (187.04) 


\section{Who's on First?}

Willis and Jozkowski (2018; see also O'Neil, Willis, \& Jozkowski, 2017) studied "linguistic sexism" in 10 social science journals, including four sex research periodicals, for articles published in 2016. They coded for reference to common gendered pairs (e.g., women and men; male and female) and reported the percentage of cases in which men were presented first. Across the three journal types (sex research, health, psychology), men were on first more often than women. Of the four sex research journals, Sex Roles was the most equitable (57.7\% "male firstness"), followed by Journal of Sex Research (74.7\%), Archives of Sexual Behavior (81.6\%), and Journal of Sex and Marital Therapy (88.8\%). This is, potentially, a nice example of unconscious/implicit bias. However, interpretation of the data is limited by the fact that Willis and Jozkowski did not consider potential confounding factors, such as the gender/ sex of the first (or senior) author and country of the researchers. It is also unclear if who is on first has any bearing on the quality of the publications and their contribution to advances in sexual science.

\section{The Hypatia Fiasco}

The practice of science is far from perfect. We are acutely aware of unethical conduct (think The Tuskegee Syphilis Experiment [Jones, 1981]) or questionable conduct. Consider, for example, the 2018 resignation of Brenda Fitzgerald as the Director of the Centers for Disease Control and Prevention after just 6 months on the job, apparently because of "...criticism of her investments with her husband in tobacco and health care companies..." (Kaplan, 2018). This gives a variant meaning to the 1978 comedy film "Up in Smoke" (think Cheech \& Chong). In modern times, Retraction Watch (https://retractionwatch.com/) provides one with a daily dose of scientist/scientific misconduct, abuse of power, retractions, etc. It costs nothing to get its daily alerts.

In 2017, there was, what I would like to call, the Hypatia fiasco, which was not a typical example of what might make its way into Retraction Watch. I will wager a bet that this feminist philosophy journal is probably not on the "content alert list" of journals of most members of the International Academy of Sex Research or readers of this Journal (see Tables 2, 3, 4, and 5). Last year, after going through peer review, Tuvel (2017) published an essay in Hypatia entitled "In Defense of Transracialism" (think Rachel Dolezal) and philosophized about the similarities and differences between "transracialism" and "transgenderism" (think Caitlyn Jenner). This did not go over well and social media went wild (see, for example, Brubaker, 2017; Dreher, 2017; Duggan, 2017; McKenzie, Harris, \& Zamudio-Suaréz, 2017; Schuessler,
2017; Singal, 2017; Walters, 2017; Winnubst, 2017; see also https://en.wikipedia.org/wiki/Hypatia_transracialism_contr oversy) as some philosophers jumped off the ivory tower down to the streets of virtual reality. It would be a bit too dramatic to label the reaction to the article's publication a form of intellectual terrorism-intellectual venom feels more accurate, with, among other things, calls to retract the article (the Editor of Hypatia refused to do so-see McKenzie et al. [2017]). Perhaps the attempt at intellectual censorship will boost Hypatia's impact factor-it could use the help. If you have time on your hands, reading about what went down, and thinking about the implications for "academic freedom," is worth the effort (for a thoughtful earlier volume on the issue, see Brubaker [2016]).

\section{Sex/Gender/Sexual Science in Everyday Life, with Apologies to Freud}

Do "mainstream" and social media cloud our perception of the salience of sex/gender/sexual science in everyday life? Maybe. In any case, not an hour goes by when there is "something" about sex/gender/sexual science on television, in print media, on social media (Facebook, Twitter, etc., etc., etc.). If I grab from my tray of news clippings, the last 10 are:

1. "Investigating sexual misconduct accusations, Arizona State suspends a physicist" (Chang, 2018)

2. "Yale student is found not guilty in a rare college rape trial" (Wang \& Weinstock, 2018).

3. "Belgian is convicted of sexism in public" (Schreuer, 2018).

4. "Resignation at Harvard over claims of abuse" (Saul, 2018).

5. "No safe space on campus to debate bathroom politics" (Kay, 2018).

6. "Danish police charge 1000 in child porn case" (Sorensen, 2018).

7. "60\% of youths say they've 'sexted"” (Bresge, 2018).

8. "Drug regimen is said to enable transgender breastfeeding" (Yeginsu, 2018).

9. "Defending a legitimate therapy: Pelvic massage, when prescribed correctly, can provide pain relief" (Rabin, 2018).

10. "Killings in Toronto's gay community anger activists" (Levin, 2018).

In case you are wondering, I don't save newspaper articles on the sex life of Donald Trump.

While Foucault is rolling over in his grave, I think about the interface between what appears in the everyday discourse about sex/gender life and the work we, as sex/gender clinicians and scientists, do: writing papers, writing grants, giving talks, 
speaking to the media, writing trade books, fretting about writing papers, fretting about writing grants, teaching sex/gender/ sexual science, fretting about teaching sex/gender/sexual science, and seeing clients in the consulting room. It is my hope that sexual science will continue to have impactful benefits for the public that we serve and that Archives will remain part of that mission.

Acknowledgements I would like to thank my colleagues at the Springer Nature office in India (Saranya Karunakaran, Kannivel Hemaletha, and Subhashini Gopal) and David Seidenfeld at the New York office in lower Manhattan for their tireless efforts in keeping things running smoothly, and to Dr. Cindy Graham, Editor of Journal of Sex Research, for the late-night sharing of trade secrets.

\section{References}

Blanchard, R. (2018). Fraternal birth order, family size, and male homosexuality: Meta-analysis of studies spanning 25 years. Archives of Sexual Behavior, 47, 1-15.

Breedlove, S. M. (2017). Prenatal influences on human sexual orientation: Expectations versus data. Archives of Sexual Behavior, 46, $1583-1592$.

Bresge, A. (2018). 60\% of youths say they've 'sexted.' National Post. Retrieved February 6, from https://www.pressreader.com/canada/ national-post-latest-edition/20180206/281672550393508.

Brotto, L. A., \& Yule, M. (2017). Asexuality: Sexual orientation, paraphilia, sexual dysfunction, or none of the above? Archives of Sexual Behavior, 46, 619-627.

Brubaker, R. (2016). Trans: Gender and race in an age of unsettled identities. Princeton, NJ: Princeton University Press.

Brubaker, R. (2017). The uproar over 'transracialism.' New York Times. Retrieved from https://www.nytimes.com/2017/05/18/ opinion/the-uproar-over-transracialism.html? mtrref=www. google.ca\&gwh=51ADB5E1717A79AFD47343BBCE5D21 $0 A \& g w t=$ pay\&assetType $=$ opinion.

Chang, K. (2018). Investigating sexual misconduct accusations, Arizona State suspends a physicist. New York Times. Retrieved March 7, from https://www.nytimes.com/2018/03/07/science/lawrencekrauss-arizona-state.html?mtrref $=$ www.google.ca\&gwh $=043 \mathrm{D} 8$ AA8450C882DBB2059BA6A971C4A\&gwt=pay.

Chivers, M. L. (2017). The specificity of women's sexual response and its relationship with sexual orientations: A review and ten hypotheses. Archives of Sexual Behavior, 46, 1161-1179.

Dreher, R. (2017). The self-murder of academic philosophy. The American Conservative. Retrieved from http://www.theamericancons ervative.com/dreher/the-self-murder-of-academic-philosophy/.

Duggan, L. (2017). Hypatia and cultures of critique. Retrieved from https://bullybloggers.wordpress.com/2017/05/06/another-day/.

Frank, K. (2018). Rethinking risk, culture, and intervention in collective sex environments. Archives of Sexual Behavior. https://doi. org/10.1007/s10508-018-1153-3.

Jones, J. H. (1981). Bad blood: The Tuskegee syphilis experiment. New York: Free Press.

Kaplan, S. (2018, March 22). AIDS researcher, a longtime government adviser, is named to lead the C.D.C. New York Times, p. A12.

Kay, B. (2018). No safe space on campus to debate bathroom politics. National Post. Retrieved March 6, from http://nationalpost.com/ opinion/barbara-kay-theres-no-safe-space-on-campus-to-debat e-bathroom-politics.

Levin, D. (2018). Killings in Toronto's gay community anger activists. New York Times. Retrieved February 12, from https://www.nytim es.com/2018/02/11/world/canada/toronto-killings-bruce-mcart hur.html?mtrref=www.google.ca\&gwh=D46D6D9C4D58E7F 1B9EF3BF1D3656EA2\&gwt=pay.

McKenzie, L., Harris, A., \& Zamudio-Suaréz, F. (2017). A journal article provoked a schism in philosophy: Now the rifts are deepening. The Chronicle of Higher Education. Retrieved from https://www. chronicle.com/article/A-Journal-Article-Provoked-a/240021.

O'Neil, A., Willis, M., \& Jozkowski, K. N. (2017). Ladies first? Not so fast: Linguistic sexism in peer-reviewed research. Poster presented at the meeting of the International Academy of Sex Research, Charleston, SC.

Rabin, R. C. (2018). Defending a legitimate therapy: Pelvic massage, when prescribed correctly, can provide pain relief. New York Times. Retrieved January 31, from https://www.nytimes.com/2018/01/31/ well/live/pelvic-massage-can-be-legitimate-but-not-in-larry-nassa rs-hands.html?mtrref=www.google.ca\&gwh=C9FAB5DD3392336 5B33967903D27CC51\&gwt=pay.

Saul, S. (2018). Resignation at Harvard over claims of abuse. New York Times. Retrieved March 6, from https://www.nytim es.com/2018/03/06/us/harvard-professor-resigns-sexual-haras sment.html?mtrref=www.google.ca\&gwh=F551D08269C298C 477B77F1D9AAA1AD2\&gwt=pay.

Schreuer, M. (2018). Belgian is convicted of sexism in public. New York Times. Retrieved March 7, from https://www.nytim es.com/2018/03/06/world/europe/belgium-sexism-fine.html?mtrre $\mathrm{f}=\mathrm{www} \cdot$ google.ca\&gwh=94D68F26847C1ADE429CAA4BB $8 \mathrm{D} 153 \mathrm{C} 4 \& \mathrm{gwt}=$ pay.

Schuessler, J. (2017, May 20). Can 'trans' apply to race? New York Times, pp. C1, 8.

Seto, M. C. (2017). The puzzle of male chronophilias. Archives of Sexual Behavior, 46, 3-22.

Singal, J. (2017). This is what a modern-day witch hunt looks like. New York Magazine. Retrieved from http://nymag.com/daily/intelligen cer/2017/05/transracialism-article-controversy.html.

Sorensen, M. S. (2018). Danish police charge 1,000 in child porn case. New York Times. Retrieved January 15, from https://www.nytim es.com/2018/01/15/world/europe/denmark-child-pornography-video .html?mtrref=www.google.ca\&gwh=1B89A3A1F1F972086636 E0FC64BFB687\&gwt=pay.

Tuvel, R. (2017). In defense of transracialism. Hypatia: A Journal of Feminist Philosophy, 32, 263-278.

Vasey, P. L. (2017). Introduction to the Special Section: The puzzle of sexual orientation: What is it and how does it work? Archives of Sexual Behavior, 46, 59-61.

Vasey, P. L., \& Zucker, K. J. (2016). Target article feature [Editorial]. Archives of Sexual Behavior, 45, 767.

Walters, S. D. (2017). Academe's poisonous call-out culture. The Chronic of Higher Education. Retrieved from https://www.chron icle.com/article/Academe-s-Poisonous-Call-Out/240016.

Walton, M. T., Cantor, J. M., Bhullar, N., \& Lykins, A. D. (2017). Hypersexuality: A critical review and introduction to the "sexhavior cycle". Archives of Sexual Behavior, 46, 2231-2251.

Wang, V., \& Weinstock, C. P. (2018). Yale student is found not guilty in a rare college rape trial. New York Times. Retrieved March 7, from https://www.nytimes.com/2018/03/07/nyregion/yale-student-notguilty-saifullah-khan.html.

Willis, M., \& Jozkowski, K. N. (2018). Ladies first? Not so fast: Linguistic sexism in peer-reviewed research. Journal of Sex Research, $55,137-145$.

Winnubst, S. (2017). Why Tuvel's article so troubled its critics. The Chronicle of Higher Education. Retrieved from https://www.chron icle.com/article/Why-Tuvel-s-Article-So/240029.

Yeginsu, C. (2018). Drug regimen is said to enable transgender breastfeeding. New York Times. Retrieved from https://www.nytim es.com/2018/02/15/health/trans gender-woman-breast-feed. 
html?mtrref=www.google.ca\&gwh=3441BDCF953179C10761 EF64FCE2CD93\&gwt=pay.

Zucker, K. J. (2002). Receiving the torch in the era of sexology's renaissance [Editorial]. Archives of Sexual Behavior, 31, 1-6.
Zucker, K. J. (2010). Reports from the DSM-V Work Group on Sexual and Gender Identity Disorders [Editorial]. Archives of Sexual Behavior, 39, 217-220.

Zucker, K. J. (2016). 2 or 5 ? [Editorial]. Archives of Sexual Behavior, 45, $1279-1288$. 